\title{
A Novel Sub-pixel Refinement Method for Fillet Weld Under Structured Light Vision
}

\section{Shengfeng Chen}

Hunan University

\section{Bing Chen}

Hunan University

Jian Liu ( $\sim$ liujian@hnu.edu.cn )

Hunan University

\section{Research Article}

Keywords: Sub-pixel refinement, Weld refinement, Directional maximum projection algorithm, Robotic welding, Structured light Vision

Posted Date: September 7th, 2021

DOI: https://doi.org/10.21203/rs.3.rs-865474/v1

License: (c) (1) This work is licensed under a Creative Commons Attribution 4.0 International License. Read Full License 


\title{
A novel sub-pixel refinement method for fillet weld under structured light vision
}

Shengfeng Chen, Bing Chen, Jian Liu* (liujian@hnu.edu.cn)

State Key Laboratory of Advanced Design and Manufacture for Vehicle Body, Hunan University, Changsha 410082,

China.

Corresponding Author: Jian Liu (liujian@hnu.edu.cn)

\begin{abstract}
Intelligent welding robots based on structured light vision are widely used in industrial production. With the demands of low cost, miniaturization and flexibility, the development of embedded structured light vision systems for seam tracking is a general trend. The core is how to efficiently and precisely position weld seam with low-configuration hardware. Sub-pixel refinement can break through the limitation of physical resolution, while also reducing hardware cost to achieve the required accuracy. To fill the gap in the sub-pixel refinement for fillet weld joint, a novel sub-pixel refinement method for fillet weld joint under structured light vision is proposed, which can sub-pixel refine the fillet weld joint under various working conditions. The main novelties of the proposed method include: (1) a novel sub-pixel refinement method for fillet weld joint by using Mean Shift, weighted least square and directional maximum projection is proposed, which is robust, universal, and accurate. (2) A directional maximum projection algorithm for refining weld is proposed for the first time. (3) The method can accurately refine fillet weld joint with low-resolution image. The proposed method is robust, universal, and accurate, and as demonstrated by the following performances: the average and maximum bias are 0.73 and 3 pixels in the accurate test, positioning accuracy rate is $100 \%$ in the test of noise-free, rusty, highly reflective and arc radiation-and-spatter working conditions. the method can be expanded to a common sub-pixel refinement method for structured light intersections through simple transformation.
\end{abstract}

Keywords: Sub-pixel refinement, Weld refinement, Directional maximum projection algorithm, Robotic welding, Structured light Vision.

\section{Declarations}

\section{Funding}

1 The Ministry of Science and Technology of the People's Republic of China (Grant No. 2017YFE0128400).

2 The Key Project of Science and Technology of Changsha (Grant No. kq1804005).

\section{Conflicts of interest}

The authors claim no conflicts of interest.

\section{Availability of data and material}

Not applicable. 


\section{Code availability}

Not applicable.

\section{Authors' contributions}

Shengfeng Chen: Conceptualization, Methodology, Software, Analysis and interpretation of data, Writing and Revising.

Jian Liu: Conception and design of the work, Reviewing and Editing, Supervision.

Bing Chen: Data curation, Investigation, Validation, Visualization.

\section{Acknowledgments}

This work is supported by the China-Japan Science and Technology Joint Committee of the Ministry of Science and Technology of the People's Republic of China (Grant No. 2017YFE0128400), and the Key Project of Science and Technology of Changsha (Grant No. kq1804005). Moreover, the authors would like to express their gratitude to the reviewers for their valuable suggestions. 


\section{Introduction}

Robotic welding is widely used in industrial production because it is high efficiency, low cost, good quality consistency and can reduce arc radiation to workers. Be that as it may, welding robots basically work in "teach and playback" mode in which they have no enough adaptability and universality, particularly in welding process. With the development of intelligent technology and corresponding hardware, it turns into an unavoidable trend to automatic, adaptable and intelligentized welding producing [1]-[4]. Weld tracking and weld positioning are the core problems in intelligent welding, which can be handled easily by sensors. Vision sensors dominate all sensors for seam tracking (arc, vision, infrared, ultrasound, and electromagnetic sensors etc.), accounting for approximately $64.7 \%$ [1].

Vision sensing method can be divided into structured light vision sensing and passive vision sensing method for seam tracking. The structured light vision sensing system is composed of a laser projector and an imaging system (monocular camera or stereo camera), which projects a structured light onto the welding assembly and positions the weld seam by recognizing the structure-light feature points. Structured light vision sensing method transforms the various weld forms into structure-light feature points, so it is robust, universal, anti-interference and has a wider range of applications [5][7].

At present, most intelligent welding robots need to be equipped with a computer for calculation and image processing. High-precision weld positioning often requires a high-configuration computer and a high-resolution image acquisition system. With the demands of low cost, miniaturization and flexibility in factories, the development of embedded structured light vision sensing systems for seam tracking is a general trend. The core problem of the embedded structured light vision sensing system is how to efficiently and precisely position weld seam with low-configuration hardware.

Sub-pixel refinement can break through the limitation of physical resolution, while also reducing hardware cost to achieve the required accuracy [8], which can consider all efficiency, accuracy and cost. The sub-pixel refinement for weld seam provides the possibility and inspiration for the development of embedded structured light vision sensing system. There is almost no research on the sub-pixel refinement of weld seam in the literature we have consulted. But sub-pixel refinement methods have been widely researched and applied in the fields of camera calibration, depth recovery and 3D reconstruction. Generally, the moment-based methods, the interpolation-based methods and the fitting-based methods are common choices for sub-pixel refinement. However, in our test, these methods have good sub-pixel refinement for structure-light images without noise, but have significant errors for structure-light images with interference and noise such as rust, high reflection, and arc radiation.

In the structured light vision system, the positioning of the fillet weld joint is transformed into the extraction of the structure-light intersection, so the fillet weld joint can be positioned by locating the structure-light intersection. To refine the fillet weld joint robustly in a factory environment, a novel sub-pixel refinement method for fillet weld joint under structured light vision is proposed. In contrast to existing approaches, the proposed method is robust, universal and high-precision, which includes the following three parts: 1) Calculating the initial direction of the structure-light stripe based on Mean Shift algorithm; 2) Refining the structure-light stripe direction and coarsely locating the sub-pixel fillet weld joint based on weighted least square method; 3) Refining fillet weld joint based on the maximum projection in light stripe direction.

This work is organized as follows: The next section starts with a discussion of related work. Sec. 3 illustrates the sub-pixel refinement for fillet weld joint under structured light vision with the proposed approach in detail. In Sec. 4, the tests and result analysis are presented. The conclusions are drawn in Sec. 5. 


\section{Related work}

In some researches on weld seam recognition and seam tracking based on structured light vision, there are a few works on sub-pixel refinement for weld seam. the gravity method is the most used sub-pixel positioning method for weld seam in structured light vision, which basic idea is to calculate the sub-pixel centerline of the structure-light stripe by using the gravity method and obtain the sub-pixel feature points based on the refined centerline [3][4][9][10]. Xueqin et al. also achieved sub-pixel level laser stripe centerline positioning through the direction template method [11]. These methods have good sub-pixel positioning accuracy for weak and noise-free working conditions, but often have wrong sub-pixel positioning for heavy noise conditions such as highly reflective, and arc radiation-and-spatter welding images. In the research of weld seam positioning based on passive vision, Hairol et al. generated the sub-pixel type of data contour by using the edge mode [12], and S. et al. presented an improved algorithm of sub-pixel edge detection based on Zernike moments [13].

In other fields, there are some systematic and comprehensive researches on sub-pixel refinement. Edward et al. presented an edge operator based on two-dimensional spatial moments [14]. The operator can be implemented for virtually any size window and has been shown to locate edges in digitized images to a twentieth of a pixel. Additionally, both theoretical and experimental noise analyses show the operator has a relatively small bias in the presence of noise. Ghosal et al. proposed a sub-pixel edge location algorithm based on Zernike moments, and it is still widely used [15]. This method used discrete Zernike moments to obtain a total of three complex masks to compute all the edge parameters for sub-pixel detection. In addition, In the research of spindle speed and feed rate on the surface finish quality of kenaf fiber-reinforced composites, M. M. Ratnam et al. detected the pin gauges using the invariant moment sub-pixel edge detection method [16]. And Huang et al. proposed a sub-pixel edge detection algorithm based on Canny-Zernike moment [17].

The fitting-based methods attempt to obtain sub-pixel location by fitting the image gray levels according to the given model. Vishvjit et al. detected edges by fitting a series of one-dimensional surfaces to each kernel and accepting the surface description which was adequate in the least square (LS) sense and had the fewest parameters [18]. Jian et al. proposed a high-accuracy edge detection algorithm at sub-pixel level by using a blurred Gaussian edge model [19]. The comparison between the moment-based approach and the interpolation-based approach shows that the method has higher accuracy, even for image data with significant noise. Kisworo et al. proposed a fitting-based method using the local energy approach and the iteration implementation [20].

The interpolation-based methods mainly include cubic interpolation method [21], linear interpolation method [22] and biharmonic spline interpolation method [23]. Steger proposed an interpolation-based method to extract lines with sub-pixel level, and then edges are detected by convolution with the first order derivative of a Gaussian kernel [24]. Chen et al. proposed a sub-pixel refinement algorithm of wear edge points based on Gauss curve approximation method [25]. There are also some other sub-pixel positioning methods: in the study of 3D shapes by scanning with a light line, Heyner L. proposed some algorithms for obtaining the skeleton of each line of light with sub-pixel accuracy based on Bezier curves, linear regression, and nonlinear regression methods [26]. In the camera calibration work, Geiger et al. proposed a sub-pixel refinement method for checkerboard corners based on gradient constraints [27].

In our test, the above mainstream methods cannot sub-pixel refine fillet weld well under structured light vision. To achieve accurate, robust and universal sub-pixel refinement, we propose a novel sub-pixel refinement method for fillet weld joint under structured light vision. This method can accurately and robustly position the sub-pixel coordinates of fillet weld joint. 


\section{Methodology}

In the structured light vision system, the positioning of the fillet weld joint is transformed into the extraction of the structure-light intersection, so the fillet weld joint can be positioned by locating the structure-light intersection. Generally, captured structure-light images often contain multiple types of noise and interference because the welding condition is a workshop environment. For example, the structure-light image of a rusty welding assembly contains rusty interference; the structure-light image of a highly reflective material welding assembly contains reflective interference; the structure-light image taken during the weld tracking contains arc radiation-and-spatter interference. Therefore, the sub-pixel refinement method for fillet weld joint needs to be robust and universal under various working conditions. Fig. 1 shows several typical fillet weld joint images under structured light vision. It can be seen that only the pixels on the structure-light stripes are useful for refining the fillet weld joint, while the rust, reflections and arc spatter in the background are all harmful information. Moment-based methods, fitting-based methods, and interpolation-based methods all refine the sub-pixel coordinates with the intensity information of all pixels in the neighborhood, so they are sensitive to these various noise and interference.

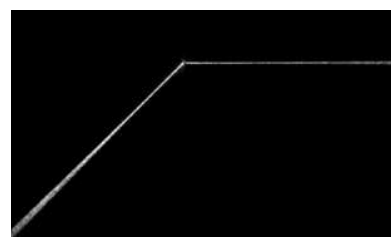

(a)

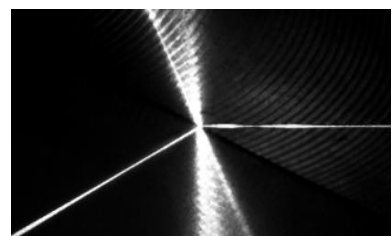

(c)

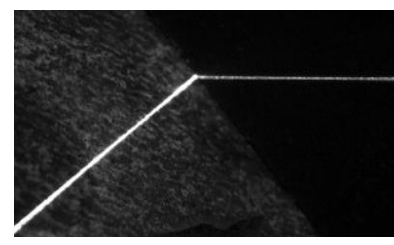

(b)

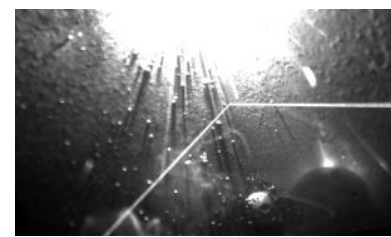

(d)

Fig. 1. Four typical fillet weld joint images under structured light vision. (a) Structure-light image without interference. (b) Structure-light image with rusty interference. (c) Structure-light image with highly reflective interference. (d) Structure-light image with arc radiation-and-spatter interference.

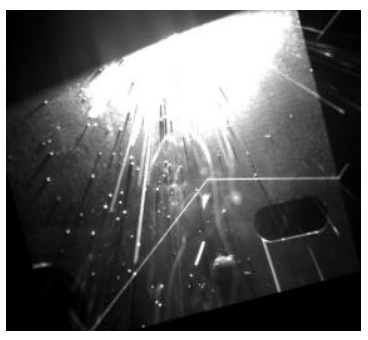

(a)

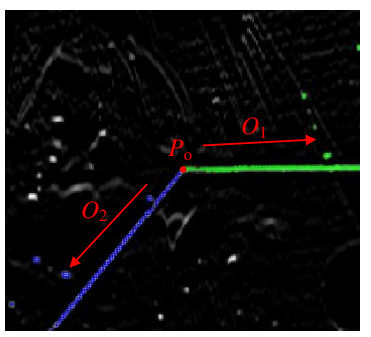

(d)

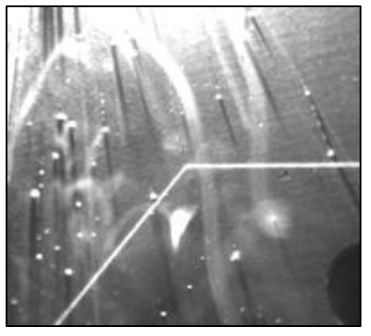

(b)

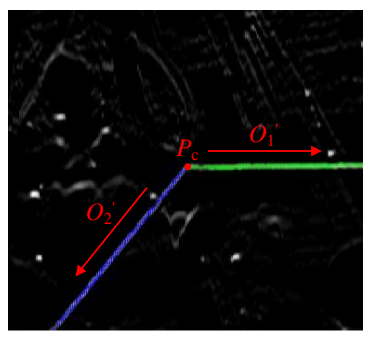

(e)

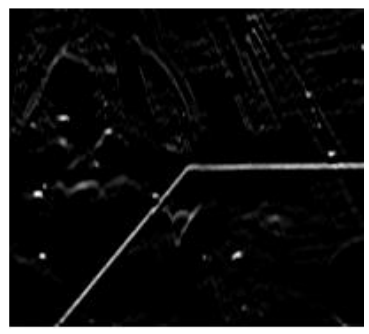

(c)

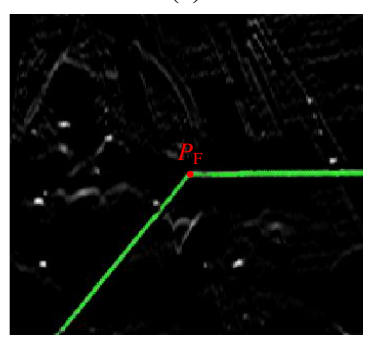

(f)

Fig. 2. Working process of the proposed sub-pixel refinement method with a structure-light image as an example. (a) 
Original image. (b) Neighborhood image. (c) Filtered image. (d) Initial direction $\overrightarrow{O_{1}}$ and $\overrightarrow{O_{2}}$ detected by Mean Shift algorithm. (e) Refined direction $\overrightarrow{O_{1}}, \overrightarrow{O_{2}}$ and coarse positioning $P_{\mathrm{c}}$ of the fillet weld joint. (f) Fine positioning $P_{F}$ based on the directional maximum projection algorithm.

Inspired by the literature [27], a novel sub-pixel refinement method for fillet weld joint under structured light vision is proposed in this paper. The proposed method refines the fillet weld joint by using pixels on the structure-light stripes, while the pixels in the background are not used, so it is robust to heavy noise. Fig. 2 shows the working process of the proposed method with a structure-light image as an example. 1) A filter kernel with linear feature enhancement is designed to remove the interference in the structure-light images as much as possible (Fig. 2 (c)). 2) The neighborhood image is converted into a matrix of angle and intensity with the initial pixel-level fillet weld joint $P_{o}$ as the center. Then, the Mean Shift algorithm is used to detect the initial direction $\vec{O}$ of the structure-light stripes (Fig. 2 (d)), where the pixels used to calculate the initial direction $\overrightarrow{O_{1}}$ and $\overrightarrow{O_{2}}$ are the green and blue pixels. 3) Refining the structure-light stripe direction $\vec{O}$ by using the weighted least square method and positioning the coarse sub-pixel coordinate $P_{c}$ of the fillet weld joint (Fig. 2 (e)). (4) For precise sub-pixel fillet weld joint $P_{F}$, the vector from $P_{F}$ to the pixels on the structure-light stripes should be approximately parallel to its corresponding refined direction $\vec{O}$. Using this fact, we proposed a sub-pixel refinement method for fillet weld joint based on the maximum projection in light stripe direction, and derive the mathematical formula of sub-pixel coordinate (Fig. 2 (f)).

\subsection{Initial direction detection of structure-light stripes based on Mean Shift algorithm}

Mean Shift algorithm is a parameter-free density estimation method, which is widely used in clustering, image smoothing, segmentation and tracking, etc. For the $n$ samples $x_{i}$ in the $d$-dimensional space $R_{d}$, the basic form of the Mean Shift vector at the $x$ is:

$$
M_{h}=\frac{1}{k} \sum_{x_{i} \in S_{h}}\left(x_{i}-x\right)
$$

where $S_{h}$ is a $d$-dimensional sphere with a radius of $h$, which satisfies the condition of $S_{h}(x)=\left\{y:(y-x)^{\mathrm{T}}(y-x) \leq h^{2}\right\}$, and $k$ is the number of samples that fall in the area $S_{h}$. Then make a new $d$-dimensional sphere with the end point of the Mean Shift vector as the center, and a new Mean Shift vector can be obtained. Repeating this step, the Mean Shift algorithm can converge to the position with the largest probability density [28][29].

In this paper, the Mean Shift algorithm in literature [28] is used to calculate the initial direction of the structure-light stripes, and the workflow is as follow:

1) Converting the neighborhood image $I_{n}$ into a matrix of angle and intensity. For the pixel coordinate $P$, the vector form $P$ to the initial pixel-level fillet weld joint $P_{o}$ is $\overrightarrow{P P_{o}}$. Then, calculating the angle $\theta(-\pi<\theta \leq \pi)$ between $\overrightarrow{P P_{o}}$ and the $x$ axis, and recording the gray level of $P$ as $S . I_{n}$ can be converted into the matrix of $\theta$ and $S$.

2) According to the intensity threshold $T_{S}$, the angle $\theta$ corresponding to the pixel $P$ that satisfy the condition of $S_{P}>$ $T_{S}$ are selected, and the data set $\operatorname{data}(\theta)$ is formed.

3) Clustering data $(\theta)$ by using the Mean Shift algorithm, and finding the center of each class. In this paper, the radius $h=\pi / 9$, the minimum distance between clusters is $h / 2$, and the threshold for end of cycle $T_{\text {stop }}=h / 100$.

4) According to the characteristics of structure-light images, the top two class centers are selected as the initial angle of the structure-light stripes. Then the initial direction $\vec{O}(\cos \theta, \sin \theta)$ are calculated (Fig. 2 (d)).

\subsection{Coarse positioning for fillet weld joint based on weighted least square method}

Due to the interference of rust, reflection, arc radiation and spatter, the clustering centers calculated in Sec. 3.1 often have a small deviation from the real structure-light stripe direction (Fig. 2(d)). It is the basis for precisely position the fillet 
weld joint in sub-pixel accuracy that obtains the precise structure-light stripe direction. To obtain more precise direction of the structure-light stripe, the weighted least square method is used to refine the structure-light stripe direction, and the coarse sub-pixel coordinates of the fillet weld joint is calculated.

The direction of the structure-light centerline is the real direction of structure-light stripe, and the intersection of centerline is the coarse sub-pixel coordinates of fillet weld joint. In this section, the weighted least squares method is used to fit the equation of the centerline. The core problem is which pixels should be selected for weighted least squares centerline fitting. It is well-known that only the pixels on the structure-light stripes are useful; but the interference in the background causes the errors of the fitting results. To avoid the interference from being selected, the weighted least squares centerline fitting formula in this paper is:

$$
\begin{array}{cl}
e=\arg \min & \sum_{P \in R_{i}} I\left(x_{i}, y_{i}\right)\left(y_{i}-k x_{i}-b\right)^{2} \\
\text { s.t. } & \cos \left\langle\overrightarrow{P_{o} P}, \overrightarrow{O_{i}}\right\rangle=\frac{\overrightarrow{P_{o} P} \times \overrightarrow{O_{i}}}{\left|\overrightarrow{P_{o} P}\right|\left|\overrightarrow{O_{i}}\right|}<0.95 \\
& \sqrt{\left|\overrightarrow{P_{o} P}\right|^{2}-\left|\overrightarrow{P_{o} P} \times \overrightarrow{O_{i}}\right|^{2}}<5 \\
& I\left(x_{i}, y_{i}\right)>T_{S}
\end{array}
$$

where $R_{i}$ is the set of pixels on the $i$ th structure-light stripe, $P=\left(x_{i}, y_{i}\right), I\left(x_{i}, y_{i}\right)$ is the gray level at pixel $P, P_{o}$ is the initial pixel-level fillet weld joint, $\overrightarrow{O_{i}}$ is the initial direction of the $i$ th structure-light stripe (calculated in Sec. 3.1), and $T_{S}$ is the intensity threshold. For a structure-light stripe, the gray level of the center pixels is large, and the gray level of the edge pixels is small. To strengthen the contribution of the center pixels to the centerline, $I\left(x_{i}, y_{i}\right)$ is used as the weighting coefficient. Three constraint conditions are used to select the pixels for structure-light centerline fitting, and their meanings are: 1$)$ the angle between the vector $\overrightarrow{P_{o} P}$ and the initial direction $\overrightarrow{O_{i}}$ needs to be less than $18^{\circ}\left(\cos 18^{\circ} \approx 0.95\right)$. 2) The distance from the pixel $P\left(x_{i}, y_{i}\right)$ to $\overrightarrow{O_{i}}$ needs to be less than 5 pixels; 3$)$ The gray level $I\left(x_{i}, y_{i}\right)>T_{S}$ at $P\left(x_{i}, y_{i}\right)$. Fig.

$\mathbf{3}$ shows the process of coarse positioning for fillet weld joint with a structure-light image as an example.

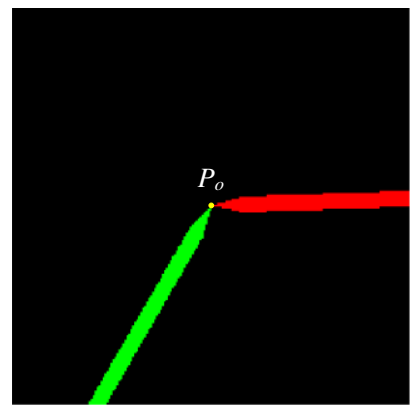

(a)

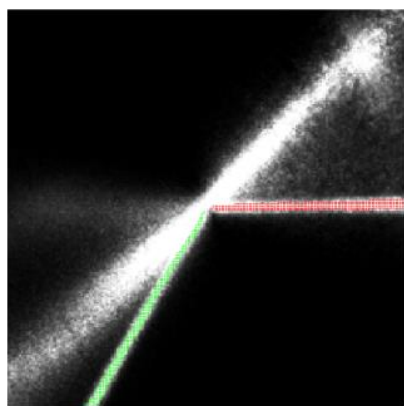

(b)

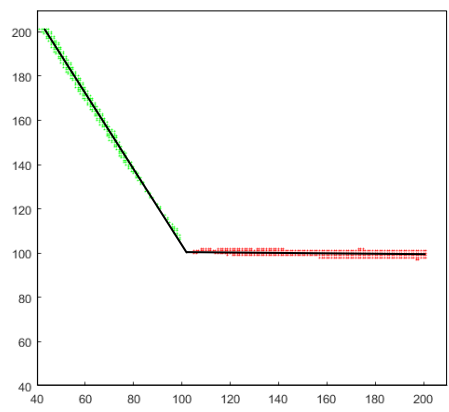

(c)

Fig. 3. The process of coarse positioning for fillet weld joint with a structure-light image as an example. (a) Graphic area corresponding to three constraint conditions in Eq. (2). (b) Select results of the pixels for structure-light centerline fitting. (c) Centerline fitting and coarse positioning based on the weighted least squares method.

Eq. (2) is straightforward to be solved in closed form (The partial derivative of $e$ is 0 at the minimum), yielding the solution:

$$
\begin{aligned}
& \frac{\partial e}{\partial k_{i}}=-2 \sum_{P \in R_{i}} I\left(x_{i}, y_{i}\right)\left(y_{i}-k_{i} x_{i}-b_{i}\right) x_{i}=0 \\
& \frac{\partial e}{\partial b_{i}}=-2 \sum_{P \in R_{i}} I\left(x_{i}, y_{i}\right)\left(y_{i}-k_{i} x_{i}-b_{i}\right)=0
\end{aligned}
$$

Arrange Eq. (3) into a matrix form: 


$$
\left[\begin{array}{cc}
\sum I\left(x_{i}, y_{i}\right) x_{i}^{2} & \sum I\left(x_{i}, y_{i}\right) x_{i} \\
\sum I\left(x_{i}, y_{i}\right) x_{i} & \sum I\left(x_{i}, y_{i}\right)
\end{array}\right]\left[\begin{array}{l}
k_{i} \\
b_{i}
\end{array}\right]=\left[\begin{array}{l}
\sum I\left(x_{i}, y_{i}\right) x_{i} y_{i} \\
\sum I\left(x_{i}, y_{i}\right) y_{i}
\end{array}\right]
$$

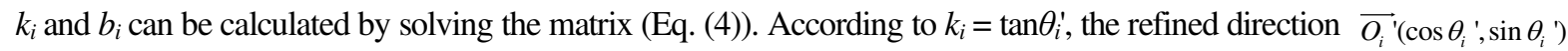
of the $i$ th structure-light stripe can be calculated. And the intersection $P_{\text {coarse }}$ of the two structure-light centerlines is the coarse positioning coordinates of the fillet weld joint.

\subsection{Fine positioning based on maximum projection in light stripe direction}

To obtain more precise sub-pixel coordinate of the fillet weld joint, we propose a sub-pixel refinement method based on maximum projection in structure-light stripe direction. As shown in Fig. 4, assuming that the theoretical fillet weld joint is located at the coordinate $P_{F}$, at neighboring pixels $P_{i j} \in R_{j}(j=1,2)$, the vector $\overrightarrow{P_{F} P_{i j}}$ should be approximately parallel to its corresponding refined direction $\overrightarrow{O_{j}}$. In reality, the width of the structure-light stripe often contains multiple pixels, especially when out of focus. Assuming that $S_{1}$ denotes the projection sum of vector $\overrightarrow{P_{F} P_{i 1}}$ on $\overrightarrow{O_{1}}$, and $S_{2}$ denotes the projection sum of vector $\overrightarrow{P_{F} P_{i 2}}$ on $\overrightarrow{O_{2}} \cdot S_{1}+S_{2}$ is the largest when $P_{F}$ is located on the actual sub-pixel fillet weld joint, leading to the optimization problem:

$$
\begin{aligned}
& P_{F}=\arg \min \sum_{j=1}^{2} \sum_{P_{i j} \in R_{j}}\left(\left|\overrightarrow{P_{F} P_{i j}}\right|^{2}-\left|\overrightarrow{P_{F} P_{i j}} \times \overrightarrow{O_{j}}\right|^{2}\right) \\
& \text { s.t. } \quad \cos \left\langle\overrightarrow{P_{\text {caarse }} P_{i j}}, \overrightarrow{O_{j}}{ }^{\prime}\right\rangle=\frac{\overrightarrow{P_{\text {causse }} P_{i j}} \times \overrightarrow{O_{j}}{ }^{\prime}}{\left|\overrightarrow{P_{\text {caarse }} P_{i j}}\right|\left|\overrightarrow{O_{j}}\right|}<0.95 \\
& \sqrt{\left|\overrightarrow{P_{\text {coarse }} P_{i j}}\right|^{2}-\left|\overrightarrow{P_{\text {coarse }} P_{i j}} \times \overrightarrow{O_{j}}\right|^{2}}<5 \\
& I\left(P_{i j}\right)>T_{S}
\end{aligned}
$$

where $P_{\text {coarse }}$ is the coarse positioning coordinates of the fillet weld joint (calculated in Sec. 3.2), $j$ represents the $j$ th structure-light stripe $(j=1,2), R_{j}$ is the pixels set on the $j$ th structure-light stripe, and $i$ is the number of pixels on the $j$ th structure-light stripe. Based on the vector theorem $\overrightarrow{P_{F} P_{i j}}=P_{i j}-P_{F}$, the solution of the problem can be transformed into the following form according to the extreme value condition (Appendix A contains the detailed derivation process of the Eq. (6)):

$$
P_{F}=\sum_{j=1}^{2} \sum_{P_{j} \in R_{j}}\left(1-\overrightarrow{O_{j}} \cdot{\overrightarrow{O_{j}}}^{\prime T}\right)^{-1} \sum_{j=1}^{2} \sum_{P_{i j} \in R_{j}}\left(1-\overrightarrow{O_{j}} \cdot{\overrightarrow{O_{j}}}^{\prime T}\right) P_{i j}
$$

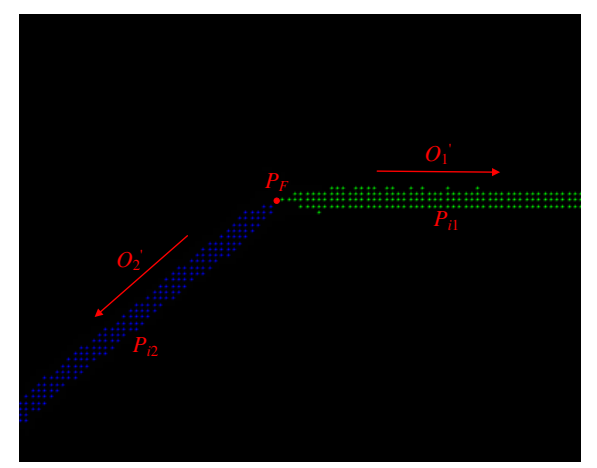

Fig. 4. The schematic diagram of the sub-pixel refinement method based on maximum projection in light stripe direction.

In order to verify the effectiveness and correctness of the sub-pixel refinement method based on maximum projection in light stripe direction, we test the algorithm by two standard images with known sub-pixel coordinates of the fillet weld joint (the coordinates of the fillet weld joint are both [101, 101]). The test result is that the sub-pixel refined fillet weld joints of the two images are both $[101,101]$. Therefore, the sub-pixel refinement method based on maximum projection in light stripe direction is effective and correct. 


\section{Test and analysis}

To evaluate our approach, the test results of accuracy, universality and robustness are shown in this section. The test hardware is a computer with a $2.8 \mathrm{GHz}$ Intel Core i5-8400 CPU, and the image processing software used is Matlab2020a. Method A (a weld refinement method based on the grayscale centroid method) [4], method B (a weld refinement method based on direction template method) [11] and method C (a universal sub-pixel refinement method based on Zernike moments) [15] are also tested to compare the sub-pixel refinement performance for fillet weld joints. It is a commonly used sub-pixel positioning method for fillet weld joints by calculating the centerline of the structure-light stripe using grayscale centroid method, and positioning the fillet weld joints according to the feature points of the centerline. Method A also detected the centerline of structure-light stripe by grayscale centroid method. To reduce noise interference, Douglas-Puke algorithm and RANSAC algorithm are used to position feature points. Method B extracted the laser stripe centerline at sub-pixel level through the direction template method, which makes the extraction accuracy of the centerline high. Method C is a universal sub-pixel refinement method based on Zernike moments, which has been widely used in image processing.

Sec. 4.1 tests the accuracy of method A, B, C and the proposed method. To quantify the accuracy, we generated six sets of structure-light images with known weld joint coordinates, and added rusty interference, highly reflective interference, and arc radiation-and-spatter interference to them. Then, we tested the deviation of the sub-pixel fillet weld joint coordinates refined by algorithm and the theoretical coordinates under the above four working conditions. Sec. 4.2 tests the universality and robustness of method A, B, C and the proposed method. Universality and robustness are directly related to the performance of the algorithm in real working conditions. For a sub-pixel refinement method with good performance, on the one hand, it should be suitable for a variety of working conditions, which corresponds to universality; on the other hand, when there is a pixel-level deviation at the initial fillet weld joint, the method can correct it during sub-pixel refinement, which corresponds to robustness.

\subsection{Accuracy test and analysis}

Accuracy is the most critical performance of the sub-pixel refinement method. Since the fillet weld joint coordinates in the structure-light image cannot be directly measured, we generated six structure-light images with known fillet weld joint coordinates by computer. The size of the six generated original images is $5000 \times 5000$ pixels, and the coordinates of the fillet weld joint in the original image are $(2501,2501)$ pixel. Then, the size of the generated original image is compressed from $5000 \times 5000$ pixels to $500 \times 500$ pixels, and the theoretical sub-pixel coordinates of the fillet weld joint in the compressed image are $(250.55,250.55)$ (because the sub-pixel range of the compressed image is $[0.5,500.5])$.

To make the generated image as similar to the real image as possible, we added rusty, highly reflective and arc radiation-and-spatter interferences to the images respectively. Finally, 24 structure-light images are generated for test: 6 structure-light images without interference, 6 structure-light images with rusty interference, 6 structure-light images with highly reflective interference, and 6 structure-light images with arc radiation-and-spatter interference. We compared the sub-pixel refinement accuracy of method A, B, C and the proposed method under four different working conditions. Fig. 5 shows a group of compressed structure-light images under four different working conditions. 


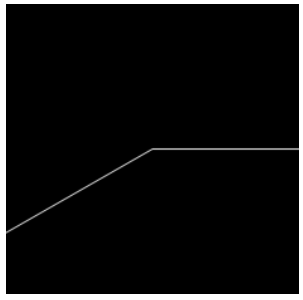

(a)

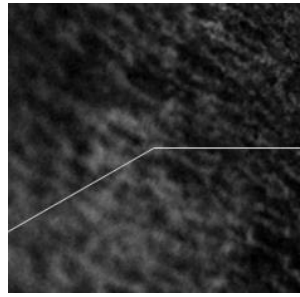

(b)

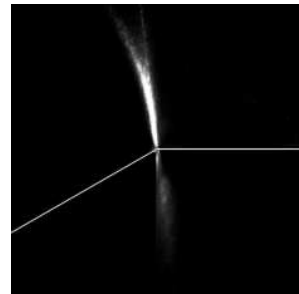

(c)

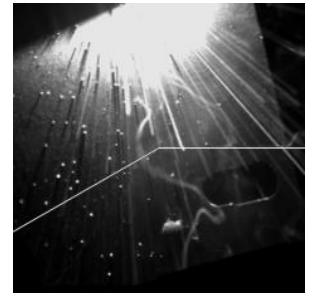

(d)

Fig. 5. Generated structure-light images. (a) Structure-light image without interference. (b) Structure-light image with rusty interference. (c) Structure-light image with highly reflective interference. (d) Structure-light image with arc radiation-and-spatter interference.

Table 1 shows the sub-pixel refinement accuracy for fillet weld joint by four different algorithms. After positioning the sub-pixel coordinates of fillet weld joint in the compressed image of $500 \times 500$ pixel size, the sub-pixel coordinates are converted to the coordinates in the original image of $5000 \times 5000$ pixels size. Then the positioning bias of the refined fillet weld joint and theoretical coordinates $([2501,2501])$ in the original image are calculated (the bias needs to be reduced by 10 times if it is calculated in the compressed image). The neighborhood image radius for sub-pixel refinement is 100 pixels in method A, B and the proposed method, and the neighborhood image radius is 7 pixels in method C.

For the structure-light images without interference, method B has the best sub-pixel positioning effect for fillet weld joint with the average bias is 0.34 pixels. Method A and the proposed method also have excellent sub-pixel positioning accuracy, but the bias of method $\mathrm{C}$ is unacceptable. For the structure-light images with rusty interference, the proposed method has the best sub-pixel positioning effect with the average and maximum biases are 0.55 pixels and 1 pixel, and method A also has acceptable sub-pixel positioning accuracy. The biases of method B and C are unacceptable. For the structure-light images with highly reflective interference, the proposed method has the best sub-pixel positioning effect with the average and maximum biases are 0.65 pixels and 1.5 pixels, but the biases of method $\mathrm{A}, \mathrm{B}$ and $\mathrm{C}$ are unacceptable. For the structure-light images with arc radiation-and-spatter interference, the proposed method has the best sub-pixel positioning effect with the average and maximum biases are 1.2 pixels and 3 pixels, but the biases of method A, $\mathrm{B}$ and $\mathrm{C}$ are unacceptable.

\section{Table 1}

Sub-pixel refinement results for fillet weld joint by four different algorithms

\begin{tabular}{|c|c|c|c|c|c|c|c|c|}
\hline \multirow{2}{*}{$\begin{array}{l}\text { Working } \\
\text { condition }\end{array}$} & \multicolumn{2}{|c|}{ Method A } & \multicolumn{2}{|c|}{ Method B } & \multicolumn{2}{|c|}{ Method C } & \multicolumn{2}{|c|}{ The proposed method } \\
\hline & $\begin{array}{c}\text { Maximum bias } \\
\text { (pixel) }\end{array}$ & $\begin{array}{c}\text { Average bias } \\
\text { (pixel) }\end{array}$ & $\begin{array}{l}\text { Maximum bias } \\
\text { (pixel) }\end{array}$ & $\begin{array}{c}\text { Average bias } \\
\text { (pixel) }\end{array}$ & $\begin{array}{c}\text { Maximum bias } \\
\text { (pixel) }\end{array}$ & $\begin{array}{c}\text { Average bias } \\
\text { (pixel) }\end{array}$ & $\begin{array}{l}\text { Maximum bias } \\
\text { (pixel) }\end{array}$ & $\begin{array}{c}\text { Average bias } \\
\text { (pixel) }\end{array}$ \\
\hline No interference & 4.5 & 2.39 & 2.3 & 0.34 & 193.8 & 77.71 & 1.2 & 0.52 \\
\hline $\begin{array}{c}\text { Rusty } \\
\text { interference }\end{array}$ & 5.5 & 2.27 & 3203.5 & 673.37 & 209.6 & 73.87 & 1 & 0.55 \\
\hline $\begin{array}{l}\text { Highly reflective } \\
\text { interference }\end{array}$ & 484.7 & 239.37 & 2701.4 & 489.16 & 174.7 & 51.6 & 1.5 & 0.65 \\
\hline $\begin{array}{l}\text { Arc-and-spatter } \\
\text { interference }\end{array}$ & 995.5 & 636.48 & 16444 & 2734.3 & 148 & 54.04 & 3 & 1.2 \\
\hline
\end{tabular}

Table 1 also reveals that method B is only useful for structure-light images without interference, because the research object in literature [11] does not include structure-light images under interference. Method $\mathrm{C}$ is useless for structure-light images under all working conditions, which is not suitable for sub-pixel refinement for fillet weld joint. Method A has a good effect on interference-free and rusty structure-light images, but is useless for heavy-interference structure-light images such as highly reflective and arc radiation-and-spatter interference. The proposed method has excellent accuracy for structure-light images under four working conditions, and it has strong anti-interference ability. Moreover, interference and noise will still reduce the accuracy of the proposed method. On the whole, the proposed method still has excellent accuracy in sub-pixel refinement for fillet weld joint, which has the potential for high-precision 
positioning for fillet weld joint with low-configuration hardware.

\subsection{Universality and robustness test}

Universality and robustness are also the core performance indicators of the fillet weld joint sub-pixel refinement method, which are directly related to the application of the sub-pixel refinement method in actual working conditions. This section will test the universality and robustness of method A, B, C and the proposed method.

\subsubsection{Universality test}

The universality of the fillet weld joint sub-pixel refinement method affects its application scope. A good sub-pixel refinement method needs to be suitable for various working conditions. This section tests the universality of method A, B, $\mathrm{C}$ and the proposed method in the working conditions of no interference, rusty interference, highly reflective interference and arc radiation-and-spatter interference. The tested images are 20 no interference, 20 rusty interference, 20 highly reflective interference, and 20 arc radiation-and-spatter interference structure-light images. Fig. 6 shows the fillet weld joint sub-pixel refinement results of method A, B, C and the proposed method under the four different working conditions.

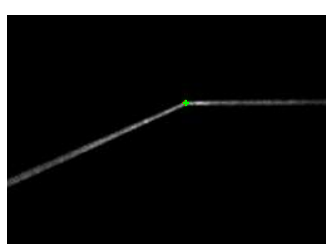

(a1)

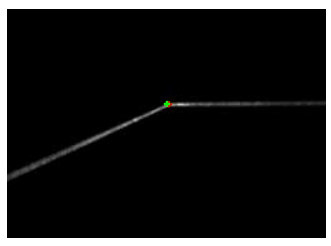

(b1)

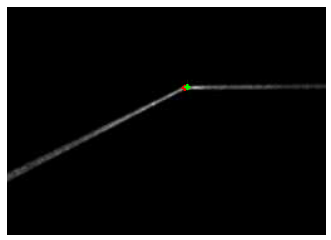

(c1)

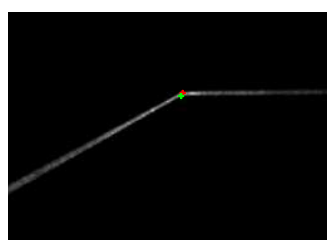

(d1)

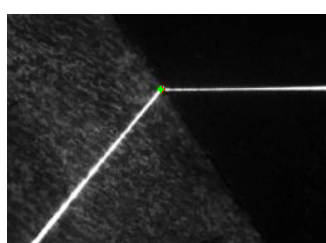

(a2)

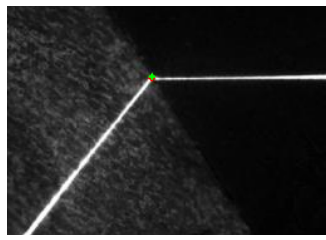

(b2)

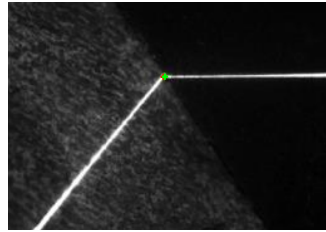

(c2)

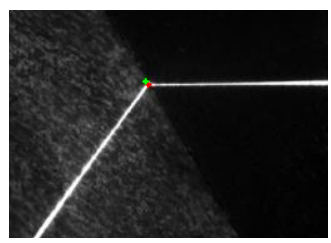

$(\mathrm{d} 2)$

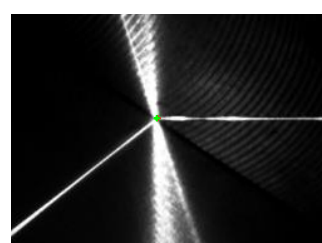

(a3)

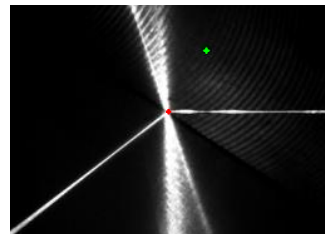

(b3)

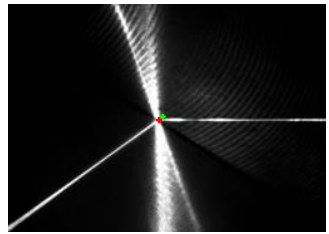

(c3)

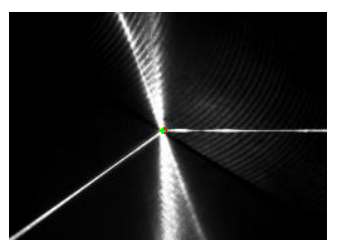

(d3)

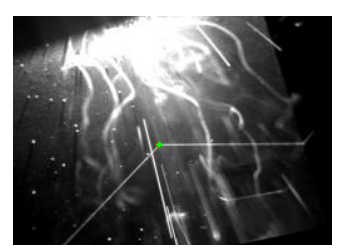

(a4)

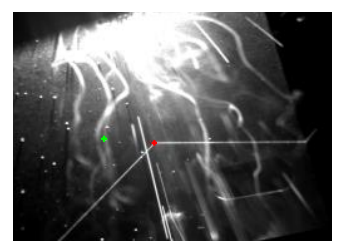

(b4)

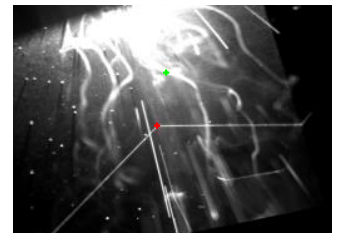

(c4)

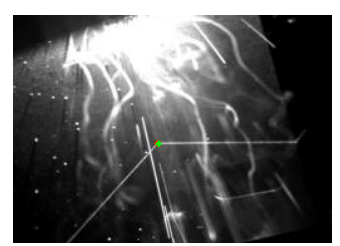

(d4)

Fig. 6. The fillet weld joint refinement results of method A, B, C and the proposed method, where red $\boldsymbol{+}$ denotes the initial pixel-level fillet weld joint coordinates, and green + denotes the sub-pixel fillet weld joint coordinates. (a1) The proposed method for structure-light images without interference. (a2) The proposed method for structure-light images with rusty interference. (a3) The proposed method for structure-light images with highly reflective interference. (a4) The proposed method for structure-light images with arc radiation-and-spatter interference. (b1) Method A for structure-light images without interference. (b2) Method A for structure-light images with rusty interference. (b3) Method A for structure-light images with highly reflective interference. (b4) Method A for structure-light images with arc radiation-and-spatter interference. (c1) Method B for structure-light images without interference. (c2) Method B for 
structure-light images with rusty interference. (c3) Method B for structure-light images with highly reflective interference. (c4) Method B for structure-light images with arc radiation-and-spatter interference. (d1) Method C for structure-light images without interference. (d2) Method C for structure-light images with rusty interference. (d3) Method C for structure-light images with highly reflective interference. (d4) Method C for structure-light images with arc radiation-and-spatter interference.

Table 2 shows the sub-pixel fillet weld joint positioning accuracy rate of method A, B, C and the proposed method under four working conditions. The positioning accuracy of fillet weld joint cannot be directly measured, so we set the criterion for judging accuracy that the deviation of the sub-pixel refinement coordinates from the initial pixel-level coordinates is less than 4 pixels, and there is no obvious visual deviation of the sub-pixel coordinates. We believe that the accuracy rate of the sub-pixel refinement method needs to reach more than $95 \%$ to meet the needs of engineering applications. Table 2 reveals that method A and B are only useful for working conditions with low interference such as no interference and rusty interference. Method $\mathrm{C}$ is useless for structure-light images under all working conditions, which is not suitable for sub-pixel refinement of fillet weld joint. The proposed method has good results for the four working conditions and has strong universality.

\section{Table 2}

Sub-pixel fillet weld joint positioning accuracy rate of method A, B, C and the proposed method

\begin{tabular}{ccccc}
\hline Working condition & The proposed method & Method A & Method B & Method C \\
\hline No interference & $100 \%$ & $100 \%$ & $85 \%$ & $55 \%$ \\
Rusty interference & $100 \%$ & $40 \%$ & $100 \%$ & $65 \%$ \\
Highly reflective interference & $100 \%$ & $20 \%$ & $35 \%$ & $65 \%$ \\
Arc-and-spatter interference & $100 \%$ & $5 \%$ & $5 \%$ & $85 \%$ \\
\hline
\end{tabular}

\subsubsection{Robustness test}

Due to the influence of noise and interference, there may be pixel-level deviations in the initial coordinates of the fillet weld joint. The deviation correction capability of the sub-pixel refinement method represents its robustness. Theoretically, the fillet weld joint is located at an accurate sub-pixel coordinates in the structure-light image (although it is difficult to accurately measure it), no matter how the initial pixel-level fillet weld joint changes, the sub-pixel coordinates refined by the sub-pixel refinement method should be located at the same position. In practice, the initial pixel-level deviation will cause the neighborhood pixels for refine the sub-pixel coordinate to change, resulting the sub-pixel coordinate of the fillet weld joint also change. For the same structure-light image, when there is a pixel-level deviation at the initial fillet weld joint, the sub-pixel coordinate variation range represents the robustness of the sub-pixel refinement method (the method is robust if the sub-pixel coordinates variation range is small). Since the universality of methods A, B and $\mathrm{C}$ does not meet the engineering requirements, it is meaningless to test their robustness. The variation range of the proposed method is tested when the initial pixel-level fillet weld joint deviates by 1 pixel, 2 pixels, 3 pixels and 4 pixels in this section.

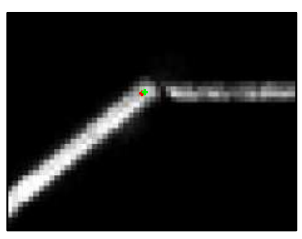

(a)

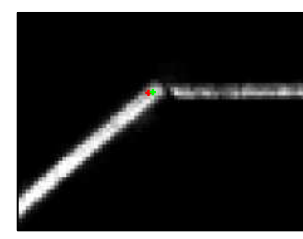

(b)

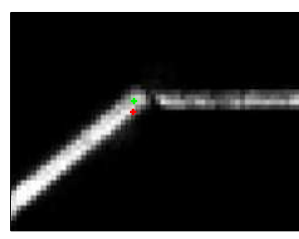

(c)

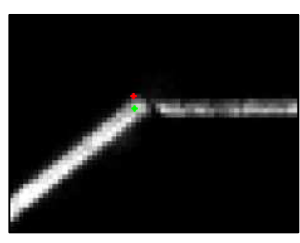

(d)

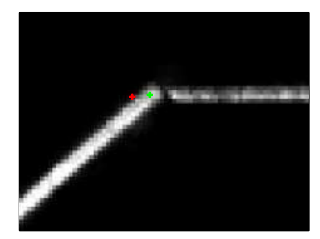

(e)

Fig. 7. The sub-pixel refinement results of the proposed method with a structure-light image as an example. (a) Sub-pixel refinement result when the initial deviation is 0 . (b) Sub-pixel refinement result when the fillet weld joint deviation is 1 
pixel. (c) Sub-pixel refinement result when the fillet weld joint deviation is 2 pixels. (d) Sub-pixel refinement result when the fillet weld joint deviation is 3 pixels. (e) Sub-pixel refinement result when the fillet weld joint deviation is 4 pixels.

Fig. 7 presents the sub-pixel refinement results of the proposed method with a structure-light image as an example. When the initial pixel-level fillet weld joint changes, the sub-pixel coordinate can always be located near the same position after sub-pixel refinement of the proposed method, which shows that the proposed method has good robustness.

Fig. 8 shows the sub-pixel refinement statistics about 20 no interference, 20 rusty interference, 20 highly reflective interference, and 20 arc radiation-and-spatter interference structure-light images. It reveals that the maximum and average deviation of the sub-pixel coordinates are 2.83 and 0.73 pixels when the initial fillet weld joint deviation does not exceed 4 pixels. For the structure-light images under the same working condition, the average deviation of the sub-pixel refinement coordinates increases as the pixel-level fillet weld joint deviation increases. The reason is that the effective neighborhood pixels used to refine the sub-pixel coordinates reduces as the initial fillet weld joint deviation increases (refer to Eq. (5)).

It also can be seen from Fig. 8 that the sub-pixel deviation variation range in the weak interference images such as no and rusty interference images is small, while the sub-pixel deviation variation range in the heavy noise images such as highly reflective and arc radiation-and-spatter interference images is large. The average deviation for highly reflective structure-light images is relatively large (up to 0.73 pixels). This is because this kind of images have multiple reflection interferences similar to structure-light stripe at the fillet weld joint (Literature [3] also supports to this result), which adds great difficulty to sub-pixel refinement. The overall test results show that the proposed method has the strong robustness and small deviation range under various working conditions.

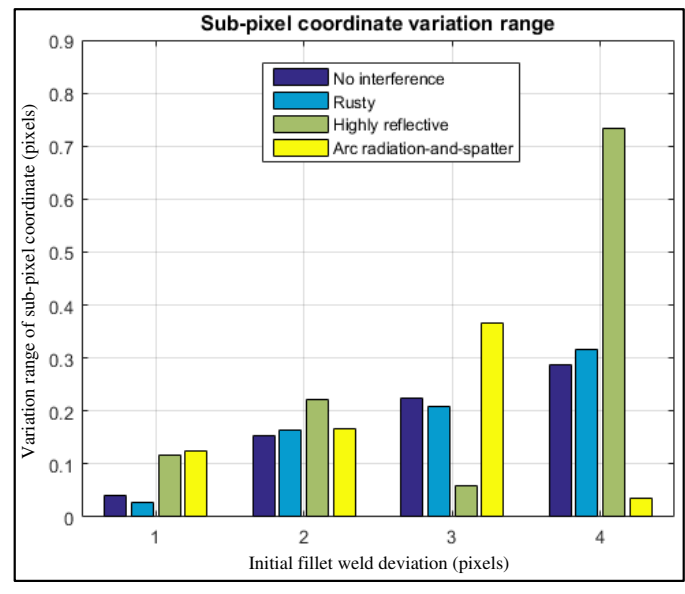

(a)

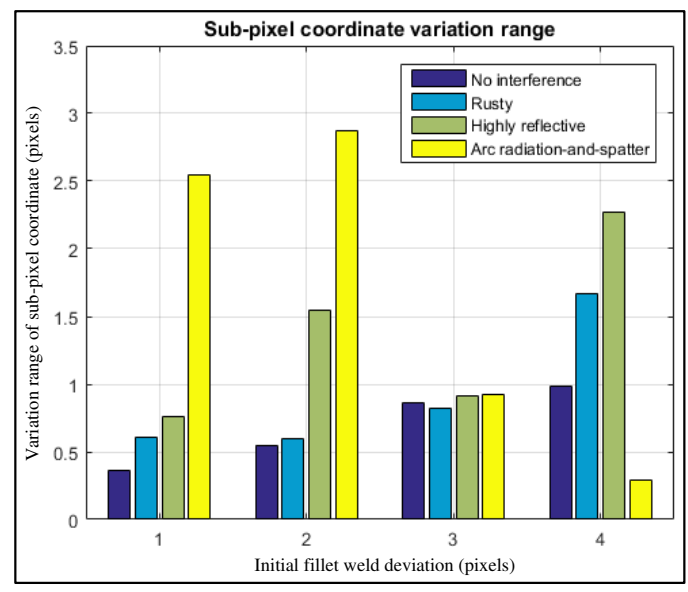

(b)

Fig. 8. The relationship between the initial fillet weld joint deviation and the deviation of sub-pixel coordinates. (a) Mean of sub-pixel coordinates deviation. (b) Maximum of sub-pixel coordinates deviation.

\section{Conclusion}

We have proposed a novel sub-pixel refinement method for fillet weld joints under structured light vision and shown its effectiveness under various working conditions. Aiming at the research gap in the sub-pixel refinement of fillet weld joint, this paper proposed a novel sub-pixel refinement method based on the fact that the cumulative sum of structure-light stripe projections is the largest on its direction vector, and deduces its mathematical formula. While our method is proposed for fillet weld joint, we believe that extending our method to a common sub-pixel refinement method for structured light intersections through simple transformation.

The method is accurate, universal, and robust, as demonstrated by the following main performance indices: 1) the 
average and maximum bias are $0.73,3$ pixels respectively in the test of generated structure-light images. 2) Sub-pixel fillet weld joint positioning accuracy rate is $100 \%$ in the test of noise-free, rusty, highly reflective and arc radiation-and-spatter structure-light images. 3) The maximum and average deviation of the sub-pixel coordinates are 2.83 and 0.73pixels when the initial fillet weld joint deviation does not exceed 4 pixels.

\section{Conflicts of interest}

The authors claim no conflicts of interest.

\section{Reference}

[1] Rout A, Deepak B, Biswal B B (2019) Advances in weld seam tracking techniques for robotic welding: A review. Robot Comput Integr Manuf 56: 12-37. https://doi.org/10.1016/j.rcim.2018.08.003

[2] Shao W J, Liu X F, Wu Z J (2019) A robust weld seam detection method based on particle filter for laser welding by using a passive vision sensor. Int J Adv Manuf Technol 104 (5): 2971-2980. https://doi.org/10.1007/ s00170-019-04029-x

[3] Shah H N M, Sulaiman M, Shukor A Z (2017) Autonomous detection and identification of weld seam path shape position. Int J Adv Manuf Technol 92 (9): 3739-3747. https://doi.org/10.1007/s00170-017-0380-4

[4] Wang N, Zhong K, Shi X. A robust weld seam recognition method under heavy noise based on structured-light vision. Robot Comput Integr Manuf 2020; 61: 101821. https://doi.org/10.1016/j.rcim.2019.101821.

[5] Yang L, Liu Y, Peng J (2020) Advances techniques of the structured light sensing in intelligent welding robots: a review. Int J Adv Manuf Technol 1-20. https://doi.org/10.1007/s00170-020-05524-2

[6] Muhammad J, Altun H, Abo-Serie E (2017) Welding seam profiling techniques based on active vision sensing for intelligent robotic welding. Int J Adv Manuf Technol 88 (1-4): 127-145. https://doi.org/10.1007/s00170-016-8707-0

[7] Zou Y, Wei X, Chen J (2020) Conditional generative adversarial network-based training image inpainting for laser vision seam tracking. Opt Laser Eng 134: 106140. https://doi.org/10.1016/j.optlaseng.2020.106140

[8] Ying-Dong Q, Cheng-Song C, et al (2005) A fast subpixel edge detection method using Sobel-Zernike moments operator. Image Vision Comput 23 (1): 11-17. https://doi.org/10.1016/j.imavis.2004.07.003

[9] Gong Y, Dai X, Li X (2010) Structured-light based joint recognition using bottom-up and top-down combined visual processing. 2010 International Conference on Image Analysis and Signal Processing 507-512.https://doi.org/ 10.1109/IASP.2010.5476064.

[10] Lee K, Hwang I, et al (2020) Real-time weld quality prediction using a laser vision sensor in a lap fillet joint during gas metal arc welding. Sensors 20 (6): 1625. https://doi.org/10.3390/s20061625

[11] Lü X, Gu D, et al (2018) Feature extraction of welding seam image based on laser vision. IEEE Sens J 18 (11): 4715-4724. https://doi.org/10.1109/JSEN.2018.2824660

[12] Shah H N M, Sulaiman M, et al (2018) Butt welding joints recognition and location identification by using local thresholding. Robot Comput Integr Manuf 52: 181-188. https://doi.org/10.1016/j.rcim.2017.12.007

[13] Chen S B, Chen X Z, et al (2005) Acquisition of weld seam dimensional position information for arc welding robot based on vision computing. J Intell Robot Syst 43 (1): 77-97. https://doi.org/10.1007/s10846-005-2966-6

[14] Lyvers E P, Mitchell O R, Akey M L (1989) Sub pixel measurements using a moment-based edge operator. IEEE T Pattern Anal 11. 12: 1293-1309. https://doi.org/10.1109/34.41367 
[15] Ghosal S, Mehrotra R (1993) Orthogonal moment operators for sub pixel edge detection. Pattern Recogn 26. 2 : 295-306. https://doi.org/10.1016/0031-3203(93)90038-X

[16] Too M X, Ratnam M M, Akil H M (2020) Investigation on the effect of machining parameters on surface roughness during turning of kenaf fiber-reinforced composite using non-contact vision method. Int J Adv Manuf Technol 110 (1): 309-325. https://doi.org/10.1007/s00170-020-05691-2

[17] Huang C, Jin W, et al (2020) Sub-Pixel Edge Detection Algorithm Based on Canny-Zernike Moment Method. J Circuit Syst Comp 29 (15): 2050238. https://doi.org/10.1142/S0218126620502382

[18] Nalwa V S, Binford T O (1986) On detecting edges. IEEE T Pattern Anal 6: 699-714. https://doi.org/10.1109/ TPAMI.1986.4767852

[19] Ye J, Fu G, Poudel U P (2005) High-accuracy edge detection with blurred edge model. Image Vision Comput 23. 5: 453-467. https://doi.org/10.1016/j.imavis.2004.07.007

[20] Kisworo M, Venkatesh S, West G (1994) Modeling edges at subpixel accuracy using the local energy approach. IEEE T Pattern Anal 16. 4: 405-410. https://doi.org/10.1109/34.277593

[21] Gallagher A C (2005) Detection of linear and cubic interpolation in JPEG compressed images. The 2nd Canadian Conference on Computer and Robot Vision. https://doi.org/10.1109/CRV.2005.33

[22] Watson D (2013) Contouring: a guide to the analysis and display of spatial data. Elsevier. https://doi.org/10.1016/ 0098-3004(93)90069-H

[23] Sandwell D T (1987) Biharmonic spline interpolation of GEOS-3 and SEASAT altimeter data. Geophys Res Lett 14. 2: 139-142. https://doi.org/10.1029/GL014i002p00139

[24] Steger C (2000) Sub pixel-precise extraction of lines and edges. Int Arch Photogr Remote Sens 33. 3: 141-156.

[25] C Zhang, J Zhang (2013) On-line tool wear measurement for ball-end milling cutter based on machine vision. Comput Ind 64. 6: 708-719. https://doi.org/10.1016/j.compind.2013.03.010

[26] Vilchez-Rojas H L, Rayas J A, Martínez-García A (2020) Use of white light profiles for the contouring of objects. Opt Laser Eng 134: 106295. https://doi.org/10.1016/j.optlaseng.2020.106295

[27] Geiger A, Moosmann F, Car Ö (2012) Automatic camera and range sensor calibration using a single shot. 2012 IEEE International Conference on Robotics and Automation. https://doi.org/10.1109/ICRA. 2012.6224570

[28] Comaniciu D, Meer P (2002) Mean shift: A robust approach toward feature space analysis. IEEE T Pattern Anal 24. 2: 603-619. https://doi.org/10.1109/34.1000236

[29] Cheng Y (1995) Mean shift, mode seeking, and clustering. IEEE T Pattern Anal 17. 8: 790-799. https://doi.org/ $10.1109 / 34.400568$

\section{Appendix A}

Assume that the direction of structure-light stripe 1 is $\overrightarrow{O_{1}{ }^{\prime}}=\left(O_{x 1}, O_{y 1}\right)$; the direction of structure-light stripe2 is $\overrightarrow{O_{2}}{ }^{\prime}=\left(O_{x 2}, O_{y 2}\right)$. Point $P_{i 1}=\left(x_{i 1}, y_{i 1}\right) \in R_{1}$ is the pixel on the structure-light stripe 1 ; point $P_{i 2}=\left(x_{i 2}, y_{i 2}\right) \in R_{2}$ is the pixel on the structure-light stripe 2. Let the coordinate of $P_{F}$ be $\left(x_{F}, y_{F}\right)$, then the formula $P_{F}=\arg \min \sum_{j=1}^{2} \sum_{P_{i j} \in R_{j}}\left(\left|\overrightarrow{P_{F} P_{i j}}\right|^{2}-\left|\overrightarrow{P_{F} P_{i j}} \times \overrightarrow{O_{j}}\right|^{2}\right)$ can be transformed into: 


$$
\begin{aligned}
f= & \sum_{P_{1} \in R_{1}}\left(\left(x_{i 1}-x_{F}\right)^{2}\left(1-O_{x 1}{ }^{2}\right)+\left(y_{i 1}-y_{F}\right)^{2}\left(1-O_{y 1}{ }^{2}\right)-2\left(x_{i 1}-x_{F}\right)\left(y_{i 1}-y_{F}\right) O_{x 1} O_{y 1}\right)+ \\
& \sum_{P_{12} \in R_{2}}\left(\left(x_{i 2}-x_{F}\right)^{2}\left(1-O_{x 2}{ }^{2}\right)+\left(y_{i 2}-y_{F}\right)^{2}\left(1-O_{y 2}{ }^{2}\right)-2\left(x_{i 2}-x_{F}\right)\left(y_{i 2}-y_{F}\right) O_{x 2} O_{y 2}\right) \\
\frac{\partial f}{\partial x_{F}}= & -2\left(1-O_{x 1}{ }^{2}\right) \sum_{P_{11} \in R_{1}}\left(x_{i 1}-x_{F}\right)+2 O_{x 1} O_{y 1} \sum_{P_{11} \in R_{1}}\left(y_{i 1}-y_{F}\right)-2\left(1-O_{x 2}{ }^{2}\right) \sum_{P_{12} \in R_{2}}\left(x_{i 2}-x_{F}\right)+2 O_{x 2} O_{y 2} \sum_{P_{12} \in R_{2}}\left(y_{i 2}-y_{F}\right)=0 \\
\frac{\partial f}{\partial y_{F}}= & -2\left(1-O_{y 1}{ }^{2}\right) \sum_{P_{11} \in R_{1}}\left(y_{i 1}-y_{F}\right)+2 O_{x 1} O_{y 1} \sum_{P_{1} \in R_{1}}\left(x_{i 1}-x_{F}\right)-2\left(1-O_{y 2}{ }^{2}\right) \sum_{P_{12} \in R_{2}}\left(y_{i 2}-y_{F}\right)+2 O_{x 2} O_{y 2} \sum_{P_{12} \in R_{2}}\left(x_{i 2}-x_{F}\right)=0
\end{aligned}
$$

Simplify and organize the above formula to get the following equation:

$$
\begin{aligned}
& {\left[n_{1}\left(1-O_{x 1}{ }^{2}\right)+n_{2}\left(1-O_{x 2}{ }^{2}\right)\right] x_{F}-\left(n_{1} O_{x 1} O_{y 1}+n_{2} O_{x 2} O_{y 2}\right) y_{F}=\left(1-O_{x 1}{ }^{2}\right) \sum_{P_{1} \in R_{1}} x_{i 1}-O_{x 1} O_{y 1} \sum_{P_{1} \in R_{1}} y_{i 1}+\left(1-O_{x 2}{ }^{2}\right) \sum_{P_{12} \in R_{2}} x_{i 2}-O_{x 2} O_{y 2} \sum_{P_{p_{2}} \in R_{2}} y_{i 2}} \\
& {\left[n_{1}\left(1-O_{y 1}{ }^{2}\right)+n_{2}\left(1-O_{y 2}{ }^{2}\right)\right] y_{F}-\left(n_{1} O_{x 1} O_{y 1}+n_{2} O_{x 2} O_{y 2}\right) x_{F}=\left(1-O_{y 1}{ }^{2}\right) \sum_{P_{1} \in R_{1}} y_{i 1}-O_{x 1} O_{y 1} \sum_{P_{11} \in R_{1}} x_{11}+\left(1-O_{y 2}{ }^{2}\right) \sum_{P_{12} \in R_{2}} y_{i 2}-O_{x 2} O_{y 2} \sum_{P_{12} \in R_{2}} x_{i 2}}
\end{aligned}
$$

Where $n_{1}$ is the pixels number in $R_{1}$, and $n_{2}$ is the pixels number in $R_{2}$. The above equation is written in matrix form as:

$$
\begin{aligned}
& {\left[\begin{array}{ll}
\sum_{j=1}^{2} n_{j}\left(1-O_{x j}{ }^{2}\right) & -\sum_{j=1}^{2} n_{j} O_{x j} O_{y j} \\
-\sum_{j=1}^{2} n_{j} O_{x j} O_{y j} & \sum_{j=1}^{2} n_{j}\left(1-O_{y j}{ }^{2}\right)
\end{array}\right]\left[\begin{array}{l}
x_{F} \\
y_{F}
\end{array}\right]=\left[\begin{array}{l}
\left(1-O_{x 1}{ }^{2}\right) \sum_{P_{1} \in R_{1}} x_{i 1}-O_{x 1} O_{y 1} \sum_{P_{11} R_{1}} y_{i 1}+\left(1-O_{x 2}{ }^{2}\right) \sum_{P_{12} \in R_{2}} x_{i 2}-O_{x 2} O_{y 2} \sum_{P_{1} \in R_{2}} y_{i 2} \\
-O_{x 1} O_{y 1} \sum_{P_{11} \in R_{1}} x_{i 1}+\left(1-O_{y 1}\right) \sum_{P_{11} \in R_{1}} y_{i 1}-O_{x 2} O_{y 2} \sum_{P_{12} \in R_{2}} x_{i 2}+\left(1-O_{y 2}{ }^{2}\right) \sum_{P_{1} \in R_{2}} y_{i 2}
\end{array}\right]} \\
& \sum_{j=1}^{2} n_{j}\left(\left[\begin{array}{ll}
1 & 0 \\
0 & 1
\end{array}\right]-\left[\begin{array}{l}
O_{x j} \\
O_{y j}
\end{array}\right]\left[\begin{array}{ll}
O_{x j} & O_{y j}
\end{array}\right]\right)\left[\begin{array}{l}
x_{F} \\
y_{F}
\end{array}\right]=\sum_{j=1}^{2}\left(\left[\begin{array}{ll}
1 & 0 \\
0 & 1
\end{array}\right]-\left[\begin{array}{c}
O_{x j} \\
O_{y j}
\end{array}\right]\left[\begin{array}{ll}
O_{x j} & O_{y j}
\end{array}\right]\right)\left[\begin{array}{c}
\sum_{P_{i j} \in R_{j}} x_{i j} \\
\sum_{P_{i j} \in R_{j}} y_{i j}
\end{array}\right]
\end{aligned}
$$

Bring $P_{i j}=\left(x_{i j}, y_{i j}\right) \in R_{j}$ and $\overrightarrow{O_{j}{ }^{\prime}}=\left(O_{x j}, O_{y j}\right)$ back to the matrix to get:

$$
\sum_{j=1}^{2} n_{j}\left(1-{\overrightarrow{O_{j}}}^{\prime}{\overrightarrow{O_{j}}}^{T}\right)\left[\begin{array}{l}
x_{F} \\
y_{F}
\end{array}\right]=\sum_{j=1}^{2} \sum_{P_{i j} \in R_{j}}\left(1-{\overrightarrow{O_{j}}}^{\prime}{\overrightarrow{O_{j}}}^{T}\right) P_{i j}
$$

Then $\left[\begin{array}{l}x_{F} \\ y_{F}\end{array}\right]=\sum_{j=1}^{2} n_{j}\left(1-\overline{O_{j}}{\overline{O_{j}}}^{T}\right)^{T} \sum_{j=1}^{-1} \sum_{P_{j} \in R_{j}}\left(1-\overline{O_{j}}{\overline{O_{j}}}^{T}{ }^{T}\right) P_{i j}$

To extend the above formula to the general form:

$$
P_{F}=\sum_{j=1}^{2} \sum_{P_{i j} \in R_{j}}\left(1-{\overline{O_{j}}}^{\prime}{\overline{O_{j}}}^{T}\right)^{T} \sum_{j=1}^{-1} \sum_{P_{i j} \in R_{j}}\left(1-{\overline{O_{j}}}^{\prime}{\overline{O_{j}}}^{T}\right) P_{i j}
$$

It is very easy to express this formula through programming. 